\title{
A MAGNITUDE INDEPENDENT SPACE-TIME EARTHQUAKE CLUSTERING ALGORITHM (MISTIC)
}

\author{
Leptokaropoulos K.M. ${ }^{1}$ and Gkarlaouni C.G. ${ }^{2}$ \\ ${ }^{1}$ Seismology and Physics of the Earth's Interior, Institute of Geophysics, Polish Academy of \\ Sciences,01-452, Warsaw, Poland, kleptoka@igf.edu.pl \\ ${ }^{2}$ Aristotle University of Thessaloniki, Department of Geophysics, 54124, Thessaloniki, Greece, \\ hara.gkarlaouni@gmail.com
}

\begin{abstract}
This paper introduces "MISTIC", a magnitude independent program for the analysis of spatio-temporal earthquake clustering, developed on the basis of a simple clustering algorithm which is implemented in Matlab. The method is conceptually based upon the comparison of the inter-event time and the epicentral/hypocentral distance between subsequent events, with pre-defined values, without any magnitude constraints. The code identifies spatial concentrations of the seismic activity which exhibit enhanced occurrence rates, significantly higher than the average or background seismicity rates. The visualization tools provided by the program's interface, enable the user to directly test and control the whole process. The calculations are performed fast even for relatively large catalogs. The description of the algorithm along with some examples are presented in this work. Moreover, an application in the local microseismicity catalog for Samos-Karaburun area, in eastern Aegean Sea, is also demonstrated. The identified clusters which are extracted from the analysis, exhibit special characteristics varying from a typical mainshock/aftershock behaviour to a swarm-like activity. Further testing and improvement of the source code are scheduled in order to constitute "MISTIC" a fast and useful tool for seismic cluster analysis.
\end{abstract}

Keywords: Seismological software, earthquake clustering, microseismicity, Samos Karaburun.

\section{Пєрí $\eta \psi \eta$}

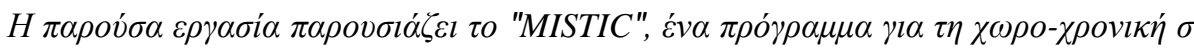

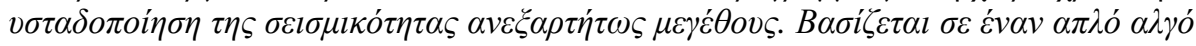

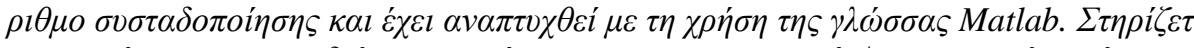

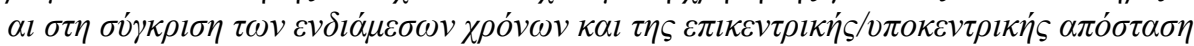

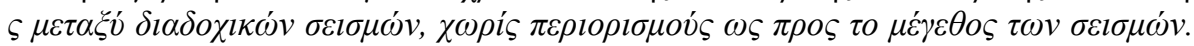

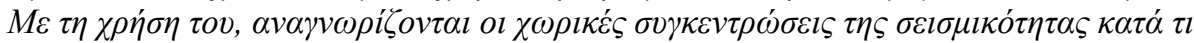

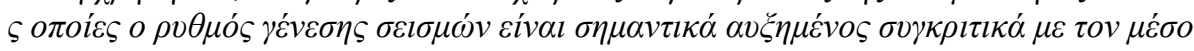


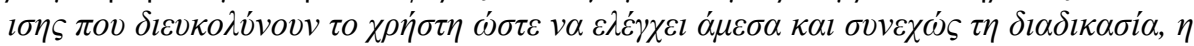

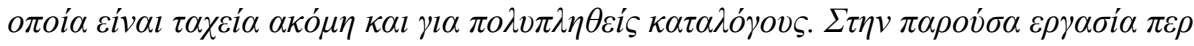

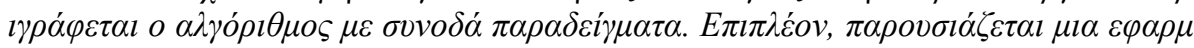

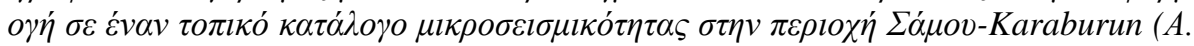

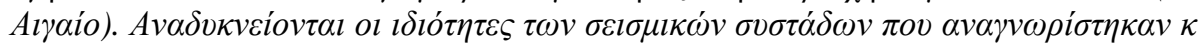




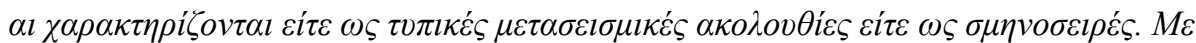

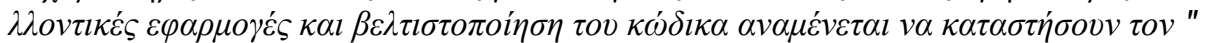

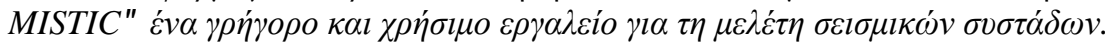

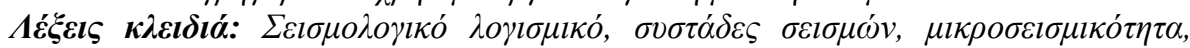
¿ános- Karaburun.

\section{Introduction}

Seismicity clustering is an aspect of earthquake behavior both for global and regional seismicity. Earthquakes exhibit multi-dimensional clustering at all scales in the sense that the earthquake occurrence, changes with respect to space, time and magnitude (e.g. Kagan and Jackson, 1991). Earthquake clustering reveals a physical interrelation mechanism among faults, or demonstrates possible interactions between earthquakes and their seismogenic sources. Clustering and earthquake interrelations stem also from the fact that some seismicity parameters (inter-event time, frequencymagnitude distribution) as well as fault properties (displacement accumulation, fault length/frequency) are characterized by fractal geometry and power law statistics. When investigating earthquake clustering in a specified seismogenic area, two seismicity types are distinguished. The first type is the time independent seismicity, which is assumed to be constant over large time periods, in a given constant fault stressing rate. Time independent seismicity is considered as normal for a certain region (Habermann and Wyss, 1984). The second kind is the triggered seismicity, such as aftershock sequences which decay with time according to Omori's law (Omori, 1894). The later type of seismicity is not representative of a region and it is oftentimes removed from the datasets when specific analysis is required. This is accomplished by the application of a seismicity declustering methodology, a process aiming to the identification and the separation of seismicity catalogs into mainshocks (independent events) and seismic excitations including foreshocks and aftershocks (dependent events).

The methods that have been developed in order to discriminate the dependent from the independent fraction of seismic activity are divided into conventional and stochastic approaches (for a comprehensive review see van Stiphout et al., 2012 and references within). The methods forming the first group can be further classified into window and link based methods. The window-based methods remove the smaller magnitude earthquakes in a space-time window around a larger event, usually named as the main shock (e.g. Utsu, 1969; Gardner and Knopoff, 1974). Most commonly, the larger the magnitude of the main shock, the bigger the window size is considered. The link-based methods remove events which are found within a compromised space-time distance compared to an earlier event (Reasenberg, 1985; Frohlich and Davis, 1990). On the other hand, stochastic declustering methods mostly model space-time-magnitude occurrences of earthquake clusters, in the form of a branching point process (e.g. Kagan, 1991; Ogata, 1998; Console and Murru, 2001; Zhuang et al., 2002, 2004). These models generally classify seismicity into two components, the background and the clustered seismicity, according to certain branching rules. However, recent studies show that the independent fraction of seismicity, as it is assumed, actually exhibits temporal fluctuations which can be noticeable even during short time scales (e.g. Hainzl and Ogata, 2005). Some modern clustering identification techniques have also been recently proposed, such as the CURATE algorithm (Jacobs et al., 2013) and multi parameter space clustering after data transformation to equivalent dimensions (Lasocki, 2014).

The aim of this paper is not to discriminate and remove aftershocks from the background seismicity, but to identify earthquake clusters in space and time without any magnitude constraints (except the completeness level of the dataset). There is strong evidence supporting the fact that the magnitude of each individual aftershock is independent of the mainshock magnitude (Michael and Jones, 1998; Felzer et al., 2004). According to this statement, the only reason that stronger earthquakes generate stronger aftershocks is because the number of their aftershocks is much larger than the one for smaller magnitude mainshocks. Additionally, seismic swarms and small seismicity bursts without characteristic events are also frequently observed in some seismotectonic environments. 
Thus, the "MISTIC" algorithm (Magnitude Independent Space - TIme Clustering) which is now introduced, is developed in order to identify clusters considering that the clustered seismicity density in space and time, is much larger than the average (or background) activity. The parameters set to classify earthquakes in clusters are the inter-event time, the distance from the cluster's center of gravity and the minimum number of events per cluster. In this way, swarms and relatively smaller magnitude seismic sequences can still be identified and thereinafter be connected with physical processes such as stress transfer and pore fluid migration. Finally, the algorithm and its application in a local seismicity catalog of Samos-Karaburun area (eastern Aegean Sea) are also demonstrated.

\section{Methodology}

"MISTIC" is a code implemented in Matlab. The cluster identification technique that it follows is based upon the calculation of characteristic quantities (time and distance) between subsequent events and the comparison of the derived values according to predefined ones, by the user limitations. The cluster analysis procedure depends on three parameters, user defined: a) a maximum inter-event time between subsequent events, b) a maximum distance between earthquakes epicentres and the clusters's gravity center and c) a minimum number of events in each cluster. This algorithm is magnitude independent, since high rated seismicity concentrated in a narrow region can be observed in the absence of a characteristic earthquake magnitude, something which is verified in the demonstrated application. A maximum inter-event time, $T_{\max }$, a maximum distance, $\mathrm{X}_{\max }$ and a minimum number of events per cluster, $\mathrm{N}_{\min }$, are assumed to be the constraints set by the user in order to define clustered activity. The source code is implemented into the following steps i) the preliminary temporal criterion, ii) the spatial criterion and iii) the final temporal criterion, all described below.

\subsection{Preliminary Temporal Criterion}

When a seismic cluster occurs, the fundamental criterion that should be fulfilled concerns the time difference between the successive events. Constraints in time are primarily considered because if an inter-event distance criterion is adopted instead, the following misleading case is likely to happen: We assume that there is a sequence of three seismic events, $\mathrm{n}_{\mathrm{i}-1}, \mathrm{n}_{\mathrm{i}}, \mathrm{n}_{\mathrm{i}+1}$ (Figure 1). Each of these events is determined by an origin time $\left(\mathrm{t}_{\mathrm{j}}\right)$ and a position vector $\left(\mathrm{r}_{\mathrm{j}}\right)$ which is described by either two variables in 2D coordinates (longitude and latitude) or three variables (plus depth) in 3D coordinates. In the current example the depth has been neglected, thus, the position vector is only defined by the epicentral coordinates.

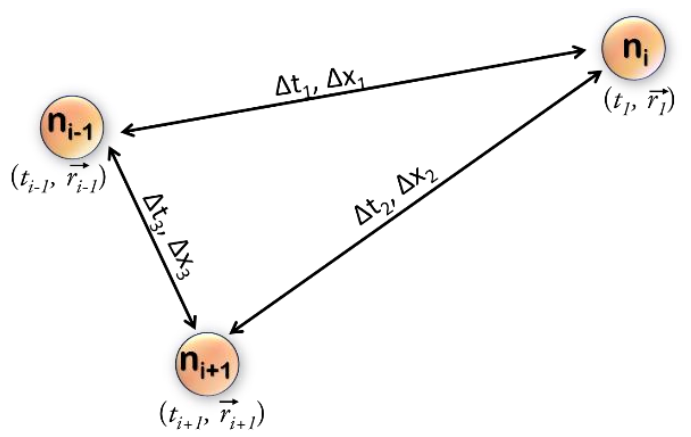

Figure 1 - An example of three earthquake epicentres in a sequence $\left(n_{i-1}, n_{i}, n_{i+1}\right)$ described by parameters in time and in 2D space, where $t_{i}$ is the occurrence time, $r_{i}$ is the position vector, $\Delta t$ is the inter-event time and $\Delta x$ is the inter-event distance between the events.

$\Delta \mathrm{T}_{\mathrm{j}}$ and $\Delta \mathrm{x}_{\mathrm{j}}$ are the temporal and spatial (Euclidian distance) differences between the successive events, respectively. In this case, a $\mathrm{n}_{\mathrm{i}}$-event although it has occurred close in time with the former $\left(\mathrm{n}_{\mathrm{i}-1}\right)$ and the following $\left(\mathrm{n}_{\mathrm{i}+1}\right)$ events $\left(\Delta \mathrm{t}_{1}, \Delta \mathrm{t}_{2}\right.$ and $\Delta \mathrm{t}_{3}$ are similar), it is located far away from them, 
such that $\Delta \mathrm{x}_{1} \gg>\Delta \mathrm{x}_{3}$ and $\Delta \mathrm{x}_{2} \gg>\Delta \mathrm{x}_{3}$. An inter-event distance criterion could classify both $\mathrm{n}_{\mathrm{i}}, \mathrm{n}_{\mathrm{i}+1}$ events out of the cluster although the distance between $n_{i+1}$ and $n_{i-1}$ is relatively much shorter. On the contrary, a predefined temporal criterion ensures that such events are not removed from the cluster. In this way, many non-clustered events in the datasets are created, however none of the clustered event is subtracted, as an outlier. After the first criterion is applied, the compiled catalogs are tested for the number of events they contain and only those for $\mathrm{N}>\mathrm{N}_{\min }$, are further investigated through the second criterion. All clusters obtained during this process are individually saved and further used as an input to the second step of the procedure.

\subsection{Spatial Criterion}

The earthquake clusters extracted after the first criterion include events with inter-event times $\Delta \mathrm{t}<\mathrm{T}_{\max }$. The second step is to set certain spatial criteria in order to define clustering in the space domain. These constraints are successively performed in two individual processes:

i. Firstly, the events whose epicentres are far from the cluster's gravity center in a distance Xi $>X_{\max }$ (specified by the user) are identified and removed. This criterion is adopted in order to manually select a radius that is in agreement with the scope and the needs of the analysis.

ii. In the case that the spatial constraints are of minor importance or not strict enough, the algorithm identifies and removes the outliers by providing the option for applying two different techniques, namely Test 1 and Test 2, which are described below:

Test 1: The first option is to identify events which lie in a distance equal or greater than the average distance $(\bar{X})$ between the events and the center of the cluster, plus $k$ times the standard deviation $(\sigma)$ of these distances from the cluster's gravity center. Specifically, the minimum acceptable distance in Test $\mathbf{1}$, where $X_{i}$ is the distance between the $i^{\text {th }}$ event and the cluster center and $k$-value is usually set equal to 2 or 3 is defined as:

Equation 1 - Minimum acceptable distance defined by Test 1.

$$
X_{i}<\bar{X}+k \sigma
$$

Test 2: The alternative option assumes that the minimum distance between two events which belong to the same cluster can be defined from the center of the cluster according to the formula:

Equation 2 - Minimum acceptable distance defined by Test 2.

$$
X_{i}<\left(\frac{\bar{X}}{X_{\max 5 \%}}\right) X+k \sigma
$$

The first term in the right part of equation 2 balances the effect of the outliers, since $\bar{X}_{\max 5 \%}$ is the average distance of the $5 \%$ of the most distant events from the center. The second term is proportional to the dispersion of the data (same as Eq. 1). If the outliers are far from the cluster gravity center $X_{\max 5 \%}$ it becomes larger and therefore, the right part decreases, such that events located at intermediate distances are also excluded. On the other hand, if the outliers are relatively close to the cluster, the term $\frac{X}{X_{\max 5 \%}}$ is closer to unity and consequently the radius extends, therefore, the events located at the borders of the cluster are not being removed. Figure 2 schematically demonstrates the comparison between the two approaches for four different combinations of input parameters (regarding $\mathrm{T}_{\max }$ and $\mathrm{X}_{\max }$ ) for the same seismic catalog. In all cases $\mathrm{N}_{\min }$ is set equal to 50 events. 

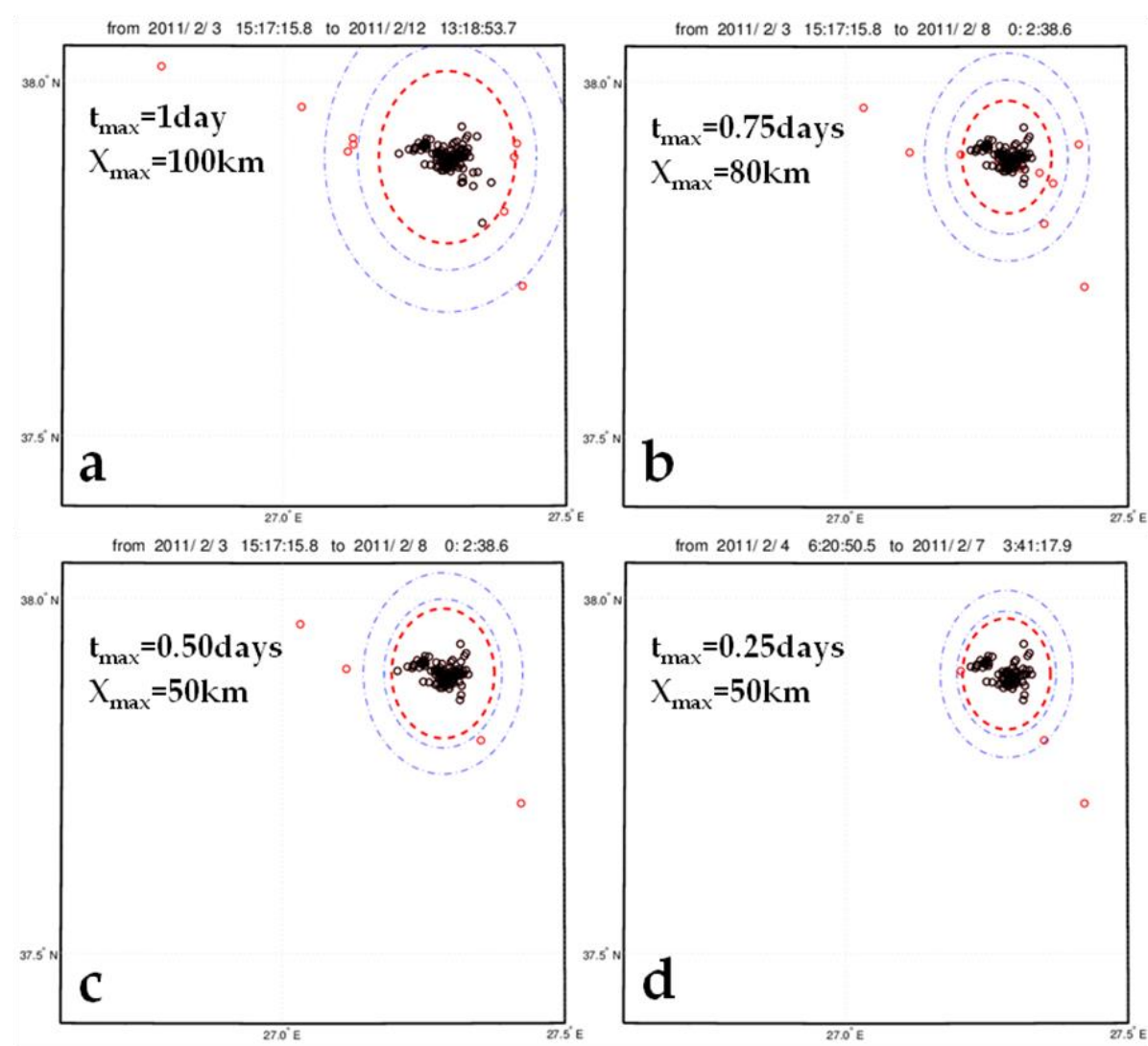

Figure 2 - Cluster identification for the same seismic dataset for different spatial and temporal criteria (Test 1 - blue circles) and (Test 2 - red circle).

In the subplots of Fig.2 the spatial distribution of earthquake epicentres which form a cluster as they have been extracted from the "MISTIC" code is shown. In each plot, the radii of the inner and the outer blue circles are equal to the mean distance of the epicentres from the cluster center plus $2 \sigma$ and $3 \sigma$, (i.e. $k=2$ and $k=3$ ) respectively (Eq. 1). Whereas, the red circle indicates the cluster boundaries defined by the term $\frac{\bar{X}}{\bar{X}_{\max 5 \%}} \bar{X}+2 \sigma$ (Eq. 2, $k=2$ ).

When $X_{\max }$ takes relatively low values, Test 2 provides almost the same constraint with the case of $2 \sigma$ although it is always stricter. As $X_{\max }$ increases and the outliers lie in greater distances, Test 2 becomes sufficiently stricter than $2 \sigma+\bar{X}$ and prevents events that are found in intermediate distances from intruding into the cluster. An alternative way to avoid such a situation is to select a reasonably low value of $\mathrm{X}_{\max }$ (and $\mathrm{T}_{\max }$ ) from the beginning of the clustering procedure so that the outliers can be initially filtered. These values can be adjusted by the user after repeating the process. In particular, in Fig. 2b, there are three events inside the red circle which have been excluded from the cluster according to the second temporal criterion described in the following section (Step 3). After the spatial constraint is accomplished the compiled catalogs are sought for the number of events they include, and only those with $\mathrm{N}>\mathrm{N}_{\min }$, are further analyzed in Step 3. 


\subsection{Final Temporal Criterion}

The datasets compiled after the application of the temporal and the spatial criteria, are further filtered with a final temporal constraint. The reason for adding a supplementary test appears because after the execution of the Spatial Criterion the removal of some remote events resulted to the increase of the inter-event times between subsequent earthquakes in the specified clusters. An additional comparison of the new inter-event times with the maximum time $\left(\mathrm{T}_{\max }\right)$ set at the beginning of the process is therefore necessary in order to ensure that the spatio-temporal criteria are strictly fulfilled.

At the end of the process, the final catalogs with the extracted clusters are available for visualization and any other processes for the inspection of the method's efficiency or for the final decision making, on behalf of the user.

The output files include all the catalogs compiled in each of the three Steps, named:

1) Dataset.c*: the first catalog extracted after the application of the Preliminary Temporal Criterion (Step 1)

2) Dataset.p*: the second catalog derived after the application of the Spatial Criteria (Step 2)

3) Fincat.b*: the final catalog (after employing Steps 1, 2 and 3)

In addition to the datasets, the program provides supplementary information about the characteristic and the statistics of the original dataset (total number of events, time span of the catalog, mean - median inter-event time and area dimensions) as well as statistical information for the derived clusters (starting and ending date/time, number of events, maximum magnitude difference, etc - see Table 1).

\section{Testing and Evaluation}

\subsection{Study Area}

In July 2007, online seismological stations were deployed in the central part of the Aegean coast in western Turkey (Inan et al., 2007). Several previous studies in this area focused on the seismotectonic properties, regarding active faults and neotectonic analysis (e.g., Bozkurt, 2001, 2003; Mountrakis et al., 2003; Çiftçi and Bozkurt, 2009), study of seismic sequences (e.g., Benetatos et al., 2006; Aktar et al., 2007), or microseismicity analysis (Tan 2013). Tan et al. (2014) installed a dense local network (July 2007 to September 2012) and analyzed the recorded seismicity for faults identification.

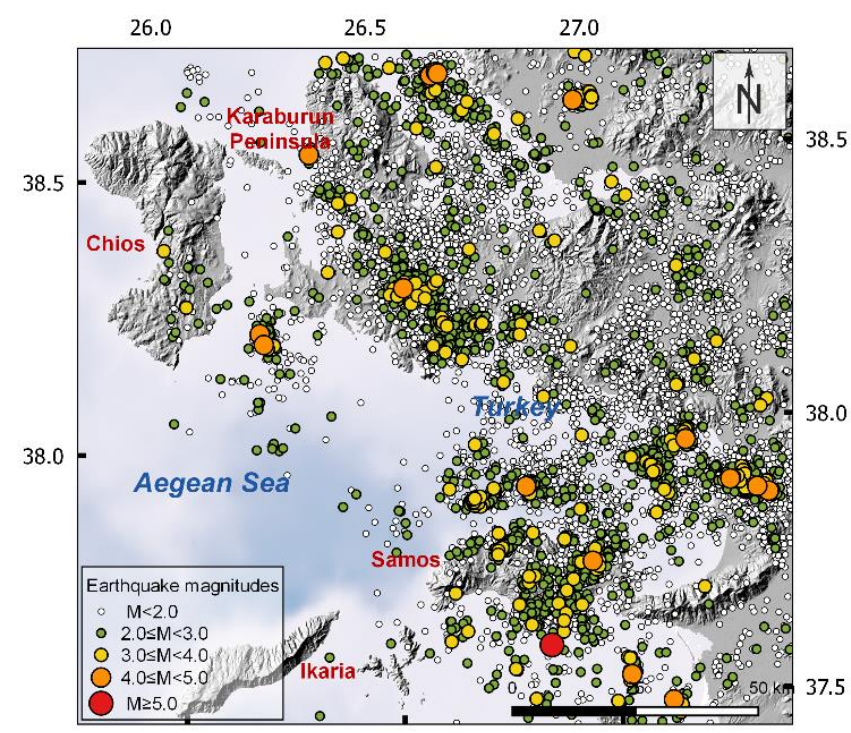

Figure 3 - Spatial distribution of the $\mathbf{1 3 . 5 9 2}$ earthquakes recorded and analysed in SamosKaraburun area. Intense seismic activity is observed at the eastern part of Samos island, NNE of Samos at the offshore Turkish mainland as well as in Karaburun peninsula. 
The earthquake foci analysis showed that they are associated with certain clusters and they illuminated the subsurface structure of the mapped faults. During this period continuous monitoring resulted in a wealth of data (Figure 3) which, along with data provided from seismological stations of the permanent Hellenic Unified Seismological Network (HUSN), are capable of revealing the geometrical properties of the activated structures. The catalog was integrated with the calculation of local magnitudes (Tan, 2013) with minimum recorded $\mathrm{M}_{\mathrm{L}}=0.2$.

The calculation of the catalog completeness magnitude, $\mathbf{M}_{\mathrm{C}}$, was accomplished by applying a maximum likelihood goodness of fit test (Leptokaropoulos et al., 2013) which is a modified version of Wiemer and Wyss (2000); $M_{C}$ was found equal to $M_{L}=1.6$ (Figure 4). The application of this technique in two years data sets shows a constant value of $M_{C}=1.6$, a fact that verifies the regular and efficient local network operation. Such a low completeness threshold implies that the specific dataset is the best catalog ever compiled for this area. The b-value of Gutenberg-Richter relation was found equal to 0.93 , a value close to 1.00 which is typical for characterizing seismic activity worldwide.
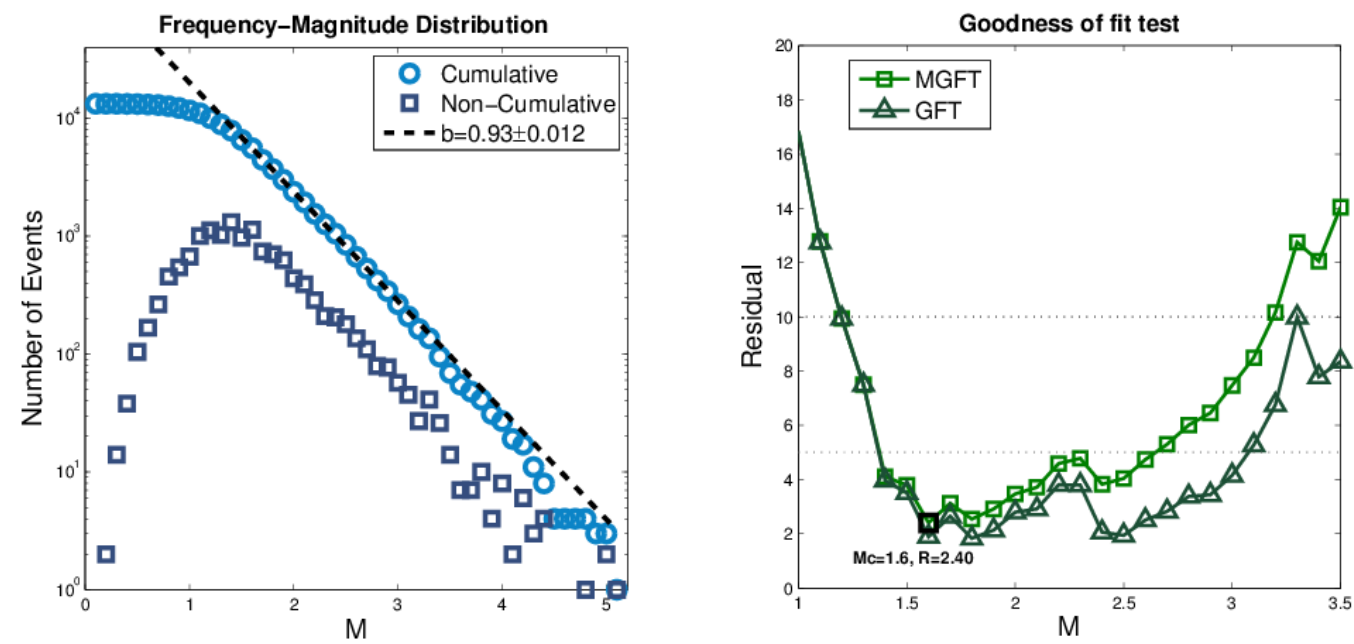

Figure 4 - Calculation of the completeness magnitude, Mc, and b-value of Gutenberg-Richter power law for the seismicity catalog. In the left frame, squares and circles indicate the incremental and the cumulative frequency magnitude distribution in respect. The fitting curve (b-value) was derived by application of the maximum likelihood estimation method. In the right frame, the goodness of fit test is shown between the obtained power laws of the real data (triangles) or 1000 synthetic catalogs (squares), as a function of magnitude, $M$.

\subsection{Application and Results}

The input seismicity dataset contains 5.577 events, distributed over an area with approximately covering $131 \mathrm{~km} \times 144 \mathrm{~km}$. The earthquake recordings lasted for 1.898 days, for which the mean and the median inter-event time is equal to 0.340 and 0.143 days respectively. The parameters used for the application of the algorithm and the extraction of earthquake clusters are i) maximum inter-event time, $T_{\max }=0.5$ days, ii) maximum distance from cluster, $\mathrm{X}_{\max }=50 \mathrm{~km}$ and iii) minimum number of events per cluster, $\mathrm{N}_{\min }=30$. Finally, 15 clusters fulfilling the aforementioned criteria, are identified. Nevertheless, two earthquake triples of these clusters are further merged into hyperclusters, i.e. clusters generated after the merge of several clusters into a single one. Although there are inter-event times greater than 0.5 days (therefore generating separated clusters), the mean inter-event time of the events included in these clusters is still much lower than the overall average. 
Thus, the analysis yields 11 clusters (Figure 5) whose properties are shown in Table 1. Some of these clusters exhibit main shock-aftershocks characteristic (C2, C5, C6, C8 and C10), whereas some others $(\mathrm{C} 1, \mathrm{C} 3, \mathrm{C} 4, \mathrm{C} 7, \mathrm{C} 9$ and $\mathrm{C} 11)$ are rather swarm-like sequences.

Table 1 - Quantitative properties of the clusters identified with their codes (the clusters are displayed in Figure 5). Shaded cells accommodate main-shock aftershock clusters, whereas cells with swarm-like clusters are white.

\begin{tabular}{|c|c|c|l|l|c|c|c|}
\hline $\begin{array}{c}\text { Cluster } \\
\text { ID }\end{array}$ & $\begin{array}{c}\text { Number } \\
\text { of Events }\end{array}$ & $\begin{array}{c}\text { Duration } \\
\text { (Days) }\end{array}$ & $\mathbf{M}_{\mathbf{L}}$ & $\mathbf{M}_{\mathbf{m a x}}$ & $\begin{array}{c}\mathbf{\Delta M} \\
\text { between the 2 } \\
\text { strongest events }\end{array}$ & $\begin{array}{c}\mathbf{M}_{\text {max }} \\
\text { Rank in } \\
\text { Sequence }\end{array}$ & $\begin{array}{c}\text { b-value } \\
\text { (95\% confidence } \\
\text { bounds) }\end{array}$ \\
\hline \hline C1 & 141 & 6.12 & 2.1 & 4.1 & 0.3 & $103 / 141$ & $0.76 \pm 0.13$ \\
\hline C2 & 37 & 2.07 & 3.1 & 4.3 & 0.9 & $2 / 37$ & $0.61 \pm 0.20$ \\
\hline C3 & 50 & 3.17 & 3.3 & 3.6 & 0.1 & $43 / 50$ & $0.75 \pm 0.21$ \\
\hline C4 & 30 & 1.48 & 2.5 & 3.3 & 0.1 & $15 / 30$ & $0.87 \pm 0.31$ \\
\hline C5 & 47 & 1.83 & 5.1 & 5.1 & 1.9 & $1 / 47$ & $0.88 \pm 0.25$ \\
\hline C6 & 69 & 4.16 & 4.8 & 4.8 & 0.6 & $1 / 69$ & $0.70 \pm 0.17$ \\
\hline C7 & 94 & 4.51 & 1.7 & 3.8 & 0.3 & $19 / 94$ & $0.80 \pm 0.16$ \\
\hline C8 & 232 & 25.79 & 1.8 & 5.0 & 0.8 & $19 / 232$ & $0.89 \pm 0.11$ \\
\hline C9 & 105 & 4.2 & 2.7 & 4.2 & 0.2 & $65 / 105$ & $1.08 \pm 0.21$ \\
\hline C10 & 97 & 5.21 & 5.0 & 5.0 & 0.6 & $1 / 97$ & $0.67 \pm 0.13$ \\
\hline C11 & 136 & 31.8 & 2.6 & 3.7 & 0.5 & $88 / 136$ & $0.73 \pm 0.13$ \\
\hline
\end{tabular}

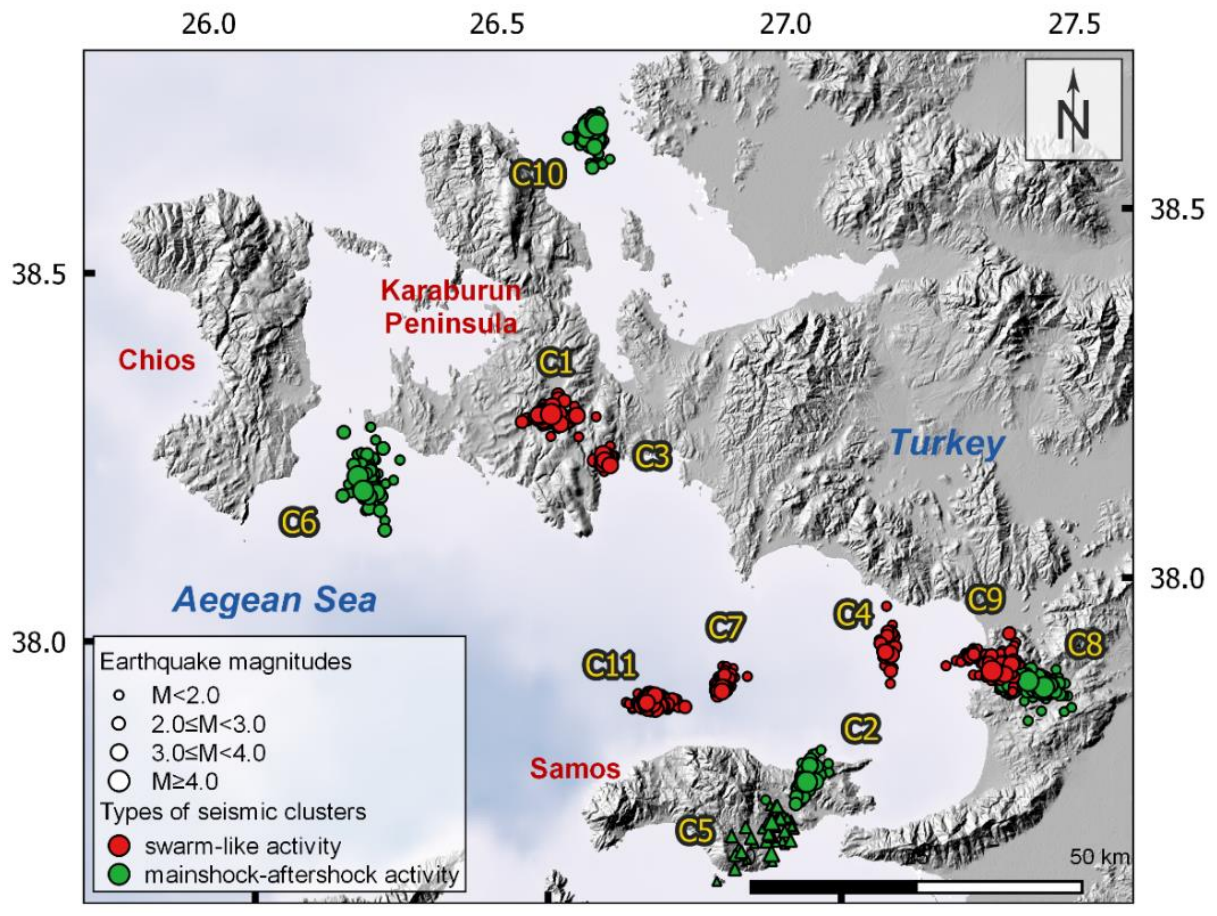

Figure 5 - The earthquake clusters identified with the use of the "MISTIC" algorithm. The cluster properties are described in Table 1. Violet colour represents a main-shock aftershock sequences, whereas red colour indicates a swarm-like activity. The usage of triangles and circles was only done for discriminating clusters from their adjacent one. 
The criteria used for this discrimination are the magnitude difference between the two strongest events ( $<0.5$ units for swarms) and the occurrence of the strongest shock at the earliest stages of the seismic burst. After several iterations, it is shown that selecting values between 0.5-1.0 days for the inter-event time, leads to an identical number and size of the extracted earthquake clusters. Lower values of inter-event time ( 0.1 to 0.5 days) yield 5-7 clusters whereas the maximum distance criterion from $30 \mathrm{~km}$ up to $\sim 80 \mathrm{~km}$ roughly increases the number of events in each cluster by $\sim 5 \%$. When the default spatial criterion is applied, a few relatively remote events are included in the clusters, in the cases when the inter-event time is set higher than one day.

\section{Discussion and Conclusion}

This paper introduced "MISTIC", a magnitude independent space-time clustering algorithm, which distinguishes seismic clusters characterized by significantly higher occurrence rates into areas with predefined dimensions. Based upon the well-established observation that seismic bursts occur when seismicity rate is more than 10 times higher than the average seismic activity (and even higher than the background seismicity), it is unlikely that the cluster's gravity center is significantly shifted from the actual location (i.e. for every 10-20 events which occur within the cluster less than one event occurs off the cluster). Especially during the initial stages of a seismic burst, when earthquake rates demonstrate the highest values, the clusters are well determined and tightly shaped, hard to be contaminated by earthquake outliers.

The application of the proposed code provides figures of clusters and lists of events potentially belonging to each cluster, for manual testing by the user. It is noteworthy that the computation time is negligible (less than $10 \mathrm{sec}$ ) even for a dataset consisting of approximately 50.000 events. This fact grants the user with the flexibility to test a variety of different parameters or value combinations and to evaluate the results along with the visualization services the code provides. The algorithm demonstrates adequate performance, being able to identify even minor clusters with the absence of a characteristic magnitude event, but only associated with the increased seismic activity. These enhanced seismicity rates may thereinafter being sought for potential association with physical processes such as pore fluid migration, dynamic or static stress changes (Leptokaropoulos et al., 2016). Nevertheless, the algorithm is not suitable for large areas or global seismicity datasets since it performs with a better accuracy at smaller areas with constrained seismic zones where high quality local catalogs are available. Further testing of the algorithm providing more results will be beneficial for the verification of the process and will certainly contribute to the optimization of the code's performance.

\section{Acknowledgments}

The authors would like to thank the editor and two anonymous reviewers for their valuable comments and suggestions. Some plots were made with the QGIS Geographic Information System, Open Source Geospatial Foundation Project (http://qgis.osgeo.org).

\section{References}

Aktar, M., Karabulut, H., Özalaybey, S. and Childs, D., 2007. A conjugate strike-slip fault system within the extensional tectonics of Western Turkey, Geophys. J. Int., 171, 1363-1375.

Benetatos, C., Kiratzi, A., Ganas, A., Ziazia, M., Plessa, A. and Drakatos, G., 2006. Strike slip motions in the Gulf of Siğaçik (western Turkey): Properties of the 17 October 2005 earthquake seismic sequence, Tectonophysics, 426, 263-279.

Bozkurt, E., 2001. Neotectonics of Turkey - a synthesis, Geodin. Acta, 14, 3-30.

Bozkurt, E., 2003. Origin of NE-trending basins in western Turkey, Geodin. Acta, 16, 61-81.

Console, R. and Murru, M., 2001. A simple and testable model for earthquake clustering, $J$. Geophys. Res., 106, 8699-8711.

Çiftçi, N.B. and Bozkurt, E., 2009. Pattern of normal faulting in the Gediz Graben, SW Turkey, Tectonophysics, 473, 234-260, doi: 10.1016/ j.tecto.2008.05.036. 
Felzer, K.R., Abercrombie, R.E. and Ekström, G., 2004. A common origin for aftershocks, foreshocks and multiplets, Bull. Seismol. Soc. Am., 94, 88-98.

Frohlich, C. and Davis, S.D., 1990. Single link cluster analysis as a method to evaluate spatial and temporal properties of earthquake catalogues, Geophys. J. Int., 100, 19-32.

Gardner, J. and Knopoff, L., 1974. Is the sequence of earthquakes in Southern California, with aftershock Removed, Poissonian? Bull. Seismol. Soc. Am., 64, 1363-1367.

Habermann, R.E. and Wyss, M., 1984. Background seismicity rates and precursory seismic quiescence: Imperial Valley, California, Bull. Seismol. Soc. Am., 74, 1743-1755.

Hainzl, S. and Ogata, Y., 2005. Detecting fluid signals in seismicity data through statistical earthquake modeling, J. Geophys. Res., 110, doi: 10.1029/2004JB003247.

Inan, S., Ergintav, S., Saatçilar, R., Tüzel, B. and İravul, Y., 2007. Turkey makes major investment in earthquake research, EOS Trans. Am. Geophys. Union, 88, 333-334.

Jacobs, K.M., Smith, E.G.C., Savage, M.K. and Zhuang, J., 2013. Cumulative rate analysis (CURATE): A clustering algorithm for swarm dominated catalogs, J. Geophys. Res. Solid Earth, 118, 553-569, doi: 10.1029/2012JB009222.

Kagan, Y.Y., 1991. Likelihood analysis of earthquake catalogues, Geophys. J. Int., 106, 135-148.

Kagan, Y.Y. and Jackson, D.D., 1991. Long-term earthquake clustering, Geophys. J. Int., 104, 117-133.

Lasocki, S., 2014. Transformation to equivalent dimensions - a new methodology to study earthquake clustering, Geophys. J. Int., 197, 1224-1235.

Leptokaropoulos, K.M., Karakostas, V.G., Papadimitriou, E.E., Adamaki, A.K., Tan, O. and İnan, S., 2013. A homogeneous earthquake catalogue compilation for western turkey and magnitude of completeness determination, Bull. Seismol. Soc. Am., 103, 5, 2739-2751.

Leptokaropoulos, K.M., Papadimitriou, E.E., Orlecka-Sikora, B. and Karakostas, V.G., 2016. An evaluation of Coulomb stress changes from earthquake productivity variations in the Western Gulf of Corinth, Greece, Pure Appl. Geophys., doi: 10.1007/s00024-015-1057-2.

Michael, A.J. and Jones, L.M., 1998. Seismicity alert probabilities at Parkfield, California, revisited, Bull. Seismol. Soc. Am., 88, 117-130.

Mountrakis, D., Kilias, A., Vavliakis, E., Psilovikos, A. and Thomaidou, E., 2003. Neotectonic map of Samos Island (Aegean Sea, Greece): Implication of Geographical Information Systems in the geological mapping. In: 4th European Congress on Regional Geoscientific Cartography and information Systems, Bologna, Italy, 11-13.

Ogata, Y., 1998. Space-time point-process models for earthquake occurrences, Ann. Inst. Stat. Math., 50, 379-402.

Omori, F., 1894. On aftershocks of earthquakes, J. College Sci. Imp. Univ., Tokyo 7, 111-200.

Reasenberg, P., 1985. Second order moment of central California Seismicity, 1969-1982, J. Geophys. Res., 90, 5479-5495.

Tan, O., 2013. The dense micro-earthquake activity at the boundary between the Anatolian and South Aegean microplates, J. Geodyn., 65, 199-217.

Tan, O., Papadimitriou, E.E., Pabucçu, Z., Karakostas, V., Yörük, A. and Leptokaropoulos, K., 2014. A detailed analysis of microseismicity is Samos and Kusadasi (Eastern Aegean Sea) areas, Acta Geophys., doi: 10.2478/s11600-013-0194-1.

Utsu, T., 1969. Aftershock and Earthquake Statistics (I): Some Parameters Which Characterize an Aftershock Sequence and Their Interrelations, J. Fac. Sci, Hokkaido Univ. Ser. VIII, Geophys., 3, 129-195.

van Stiphout, T., Zhuang, J. and Marsan, D., 2012. Seismicity declustering, Community Online Resource for Statistical Seismicity Analysis, doi: 10.5078/corssa-52382934.

Wessel, P. and Smith, W.H.F., 1998. New, improved version of the Generic Mapping Tools Released, EOS Trans. AGU, 79, 579 pp.

Wiemer, S. and Wyss, M., 2000. Minimum magnitude of completeness in earthquake catalogues: Examples from Alaska, the Western United States, and Japan, Bull. Seismol. Soc. Am., 90, 4, 859-869.

Zhuang, J., Ogata, Y. and Vere-Jones, D., 2002. Stochastic declustering of space-time earthquake occurrences, J. Am. Stat. Assoc., 97, 369-380.

Zhuang, J., Ogata Y. and Vere-Jones, D., 2004. Analyzing earthquake clustering features by using stochastic reconstruction, J. Geophys. Res., 109, B05301, doi: 10.1029/2003JB002879. 